\title{
Performance comparison of SVM and ANN for aerobic granular sludge
}

\author{
Nur Sakinah Ahmad Yasmin ${ }^{1}$, Norhaliza Abdul Wahab ${ }^{2}$, Aznah Nor Anuar ${ }^{3}$, Mustafa Bob $^{4}$ \\ ${ }^{1,2}$ School of Electrical Engineering, Faculty of Engineering, Universiti Teknologi Malaysia, Skudai, Johor, Malaysia \\ ${ }^{3}$ Department of Environment and Green Technology, Malaysia-Japan International Institute of Technology, Malaysia \\ ${ }^{4}$ Taibah University, College of Engineering, Department of Civil Engineering, Madinah, Kingdom of Saudi Arabia
}

\begin{tabular}{l} 
Article Info \\
\hline Article history: \\
Received Mar 29, 2019 \\
Revised Jun 3, 2019 \\
Accepted Jun 26, 2019 \\
\hline Keywords: \\
Aerobic granular sludge \\
ANN \\
SVM \\
SBR \\
WWTP
\end{tabular}

(1)

\begin{abstract}
To comply with growing demand for high effluent quality of Domestic Wastewater Treatment Plant (WWTP), a simple and reliable prediction model is thus needed. The wastewater treatment technology considered in this paper is an Aerobic Granular Sludge (AGS). The AGS systems are fundamentally complex due to uncertainty and non-linearity of the system makes it hard to predict. This paper presents model predictions and optimization as a tool in predicting the performance of the AGS. The input-output data used in model prediction are (COD, TN, TP, AN, and MLSS). After feature analysis, the prediction of the models using Support Vector Machine (SVM) and Feed-Forward Neural Network (FFNN) are developed and compared. The simulation of the model uses the experimental data obtained from Sequencing Batch Reactor under hot temperature of $50^{\circ} \mathrm{C}$. The simulation results indicated that the SVM is preferable to FFNN and it can provide a useful tool in predicting the effluent quality of WWTP.
\end{abstract}

Copyright $(2019$ Institute of Advanced Engineering and Science. All rights reserved.

\section{Corresponding Author:}

Norhaliza Abdul Wahab,

School of Electrical Engineering, Faculty of Engineering,

Universiti Teknologi Malaysia, 81310 Skudai Johor, Malaysia.

Email: aliza@fke.utm.my

\section{INTRODUCTION}

Wastewater is the combination of the water or liquid carried trashes removed from residential area, commercial and industrial wastes together with groundwater and surface water as may be present. This wastewater is then treated at domestic wastewater treatment system which also known as wastewater treatment plant (WWTP). About (99\%) ninty-nine percent water by weight and is basically reffered to as influent as it enters the WWTP facilty. The wastewater has three stages namely as mechanical treatment (primary treatment), biological treatment (secondary treatment) and chemical treatment (tertiary treatment). Wastewater flow been treated through all the stages of treatment before it is allowing to convey back to the consumer. This treated water is generally reffered as effluent as it flows out the WWTP facility. Wastewater treatment system involved complex process and exhibit nonlinear behaviour that resulted in time consuming and effort to develop detailed theoretical dynamic models. The main challenge of modelling and control of an AGS is it involved complexity of physical, biological and chemical treatment as it is involved with internal interactions between process variable and sludge characteristics. Most of the current available models (mathematical models) are complex for the system.

In the past, some deterministic methods such as activated sluge models (ASM1, ASM2, and ASM3) were developed to describe the mass balance of biomass (microbiology) activities in treatment process [1]. There is an assumptions made in these mathematical models mainly based on engineering principles such that models may be highly effective for the prediction of nitrogenous substrate removal and carbonaceous in waswater treatment plant system. However, due to these models are high dimensional and contain an 
outsized number of stoichiometric and kinetic parameters that should be determined by specialist process operation, they may not be practical for on-line plant control. Besides, it may not provide good prediction of plant if other current process such as sedimentation are not included in the overall plant evaluation.

A popular Artificial Intelligent (AI) such as artificial neural network (ANN) methods has been widely used for prediction for WWTP due to its good capability in modelling and prediction of process performance [2]. However, compared with multiple linear regression or other conventional methods [3-5], ANN methods shows high accuracy but large number of data is needed as some shortcomings will appeare from its theoretical statical basis for instance accuracy of the prediction is not satisfying when the training data set is small. As in against Support Vector Machine (SVM), has been proposed to solve this type of problems as SVM is based on statistical learning theory [6]. SVM has several merits compared to ANN such as efficient utilization of high-dimensional feature space, distinctively in solvable optimization problem and has a good ability in theoretical analysis using computional learning theory [4].

As a highly approach for the model with limited training samples set, the SVM has been applied in many field such prediction [7], patern recognition problem [8], regression [9, 10], classification [11] and time series analysis $[11,12]$. In addition, SVM has been reported outperformed traditional statistical learning methods such as ANN. Thus, more researcher are focusing on this method as SVM has been arousing more. For example, in order to make a beginner learner to understand SVM regression and classification were reviewed by Smola et al. [13] and Burges et al. [14], respectively. In addition, a library for SVM has been develop by [15] in order to help beginners to easily apply SVM according to their field of application. Furthermore, Noble described the explanation of SVM and its biological applications [16]. Specifically, Xu et al. adopted SVM for chemometrics in term of classification [17]. While Jia. et al. showed that SVM was able to predict the synthesis characteristic of hydraulic valve in industrial production [8]. Other than that, Liang et al. had done an effective approach on SVM for content based on sketch retrival [18]. Evaluation of using various method such as wavelets, PCA and SVM has been proposed by Gumus et al [19]. All these studies show all the application and had make a good contribution of SVM.

The example of implementation of SVM with limited dataset in WWTP can be found in [20], which developed SVM model to study the relationship between membrane bioreactor and affecting factors that cause fouling. The study reported that SVM model can assure a good prediction result compared to RBFNN model since the depending of the training dataset for SVM is lesser than RBFNN. Other than that, W. Li Juan et al. [21] done a research on predicting effluent quality of WWTP plant with cyclic activated sludge process (ASP) using SVM model. The SVM model was reported to gives a high accuracy in predicting quality effluent with small learning ability and have a good generalization. X. Xi et al. [22], presented SVM model that was applied to predict the permeate flux and rejection of Bovine serum albumin (BSA). The result shows SVM model more accurate than ANN model. This is due to ANN model need to rely on the ones expertise and experience while SVM is based on statistical theory. K. Gao et al. [23], developed SVM model for membrane permeate flux during dead-end microfiltration of activated sludge reactor. The SVM model were then compared with BPNN model shows SVM model obtained higher accuracy than BPNN. The researcher concluded that in small sample size data, SVM yield better accuracy than BPNN model. This is because SVM has a rigorous foundation either in mathematical or theoretical and it based on statistical theory despite BPNN that needs to rely on the designer knowledge. Hence, that's make SVM model only need small number of data to achieved higher prediction performance. Therefore, this paper compares the SVM and FFNN methods in term of their accuracy performance of the model using a limited experimental dataset of SBR aerobic granular sludge nutrient removal process. The aim of this paper is to minimize the correlation and mean squeare error of the training dataset. The 60 days' data (with 21 samples) under the temperature of $50 \mathrm{oC}$ is utilized to develop the model for AGS.

This paper consists of five sections and are organized as follows. The first section will introduce on background study and relavent literarature on previous study on the proposed method. The second section will be presented SVM and FFNN structure with the flow of the modelling method. The detailed of the experimental setup of this study is explained in the third section. In the fouth section is the result and discussion. The result is compared and discussed in this section. The fifth section gives conclusion and some future works.

\section{EXPERIMENT SETUP}

Experiment setup in this study were carried out in three double-walled cylindrical column bioreactor with internal diameter of $6.5 \mathrm{~cm}$ and height of $100 \mathrm{~cm}$. The sequencing bioreactor sequence (SBR) such as aerator pump, influent feeding and effluent discharge were controlled by programmable logic controller (PLC). The working temperature for the bioreactor was set to 30,40 and $50 \pm 1{ }^{\circ} \mathrm{C}$ and controlled using thermostat and water bath sleeves. The schematic diagram of the AGS system is shown in Figure 1. 


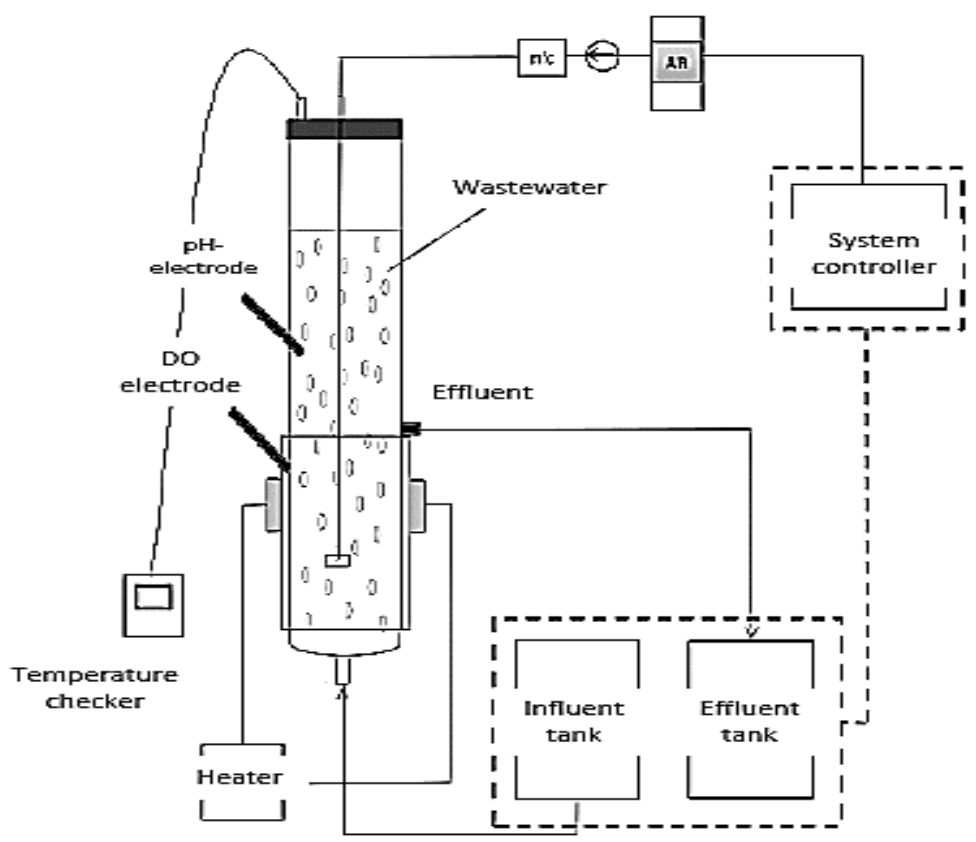

Figure 1. Schematic diagram of laboratory scale aerobic granular sludge bioreactor

In this work, the SBR operated at $50 \pm 1{ }^{\circ} \mathrm{C}$ is considered. The influent parameters that used as input of the model are chemical oxygen demand (COD), total nitrogen (TN), total phosphorus (TP), amnonia nitrogen (AN) and mixed liquor suspended solid (MLSS) while the same influent parameters are considered as an effluent or output of the model (COD, TN, TP, AN and MLSS). Figure 2 shows (a) Total Phosphorus (TP), Total Nitrogen (TN), and Amonia Nitrogen (AN) influent dataset, (b) Total Phosphorus (TP), Total Nitrogen (TN), and Amonia Nitrogen (AN) effluent dataset, (c) Chemical Oxygen Demand (COD) concentration dataset, (d) Mixed Liquor Suspended Solid (MLSS) dataset.

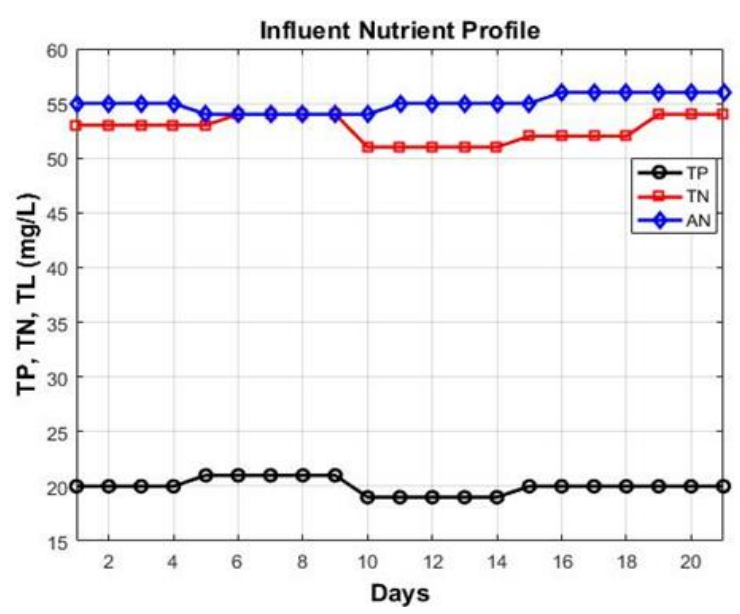

(a)

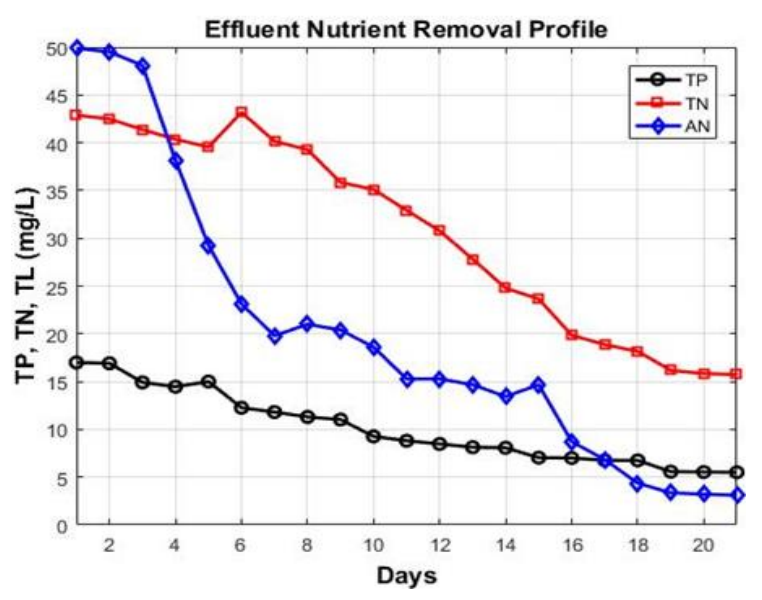

(b) 


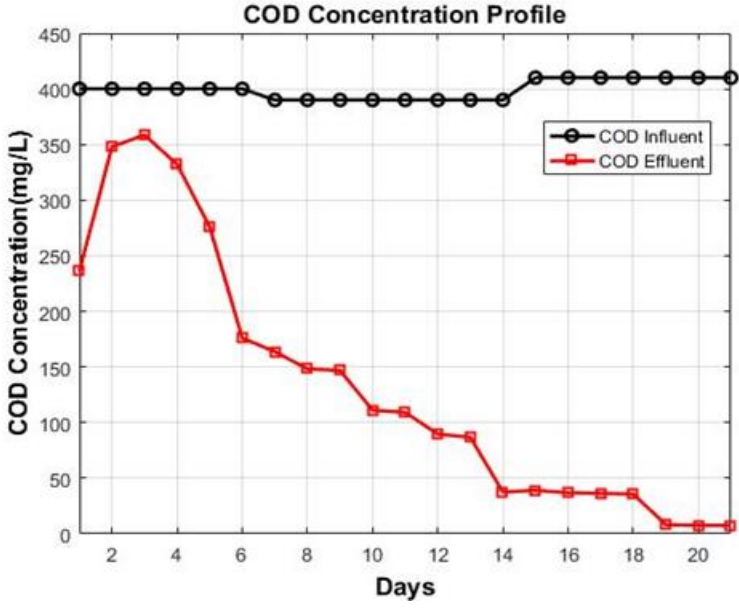

(c)

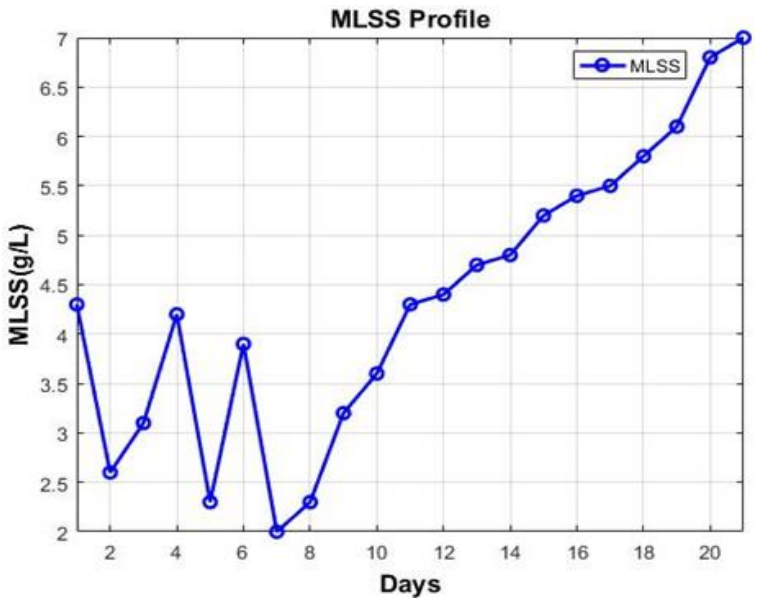

(d)

Figure 2. (a) Total Phosphorus (TP), Total Nitrogen (TN), and Amonia Nitrogen (AN) influent dataset, (b) Total Phosphorus (TP), Total Nitrogen (TN), and Amonia Nitrogen (AN) effluent dataset, (c) Chemical Oxygen Demand (COD) concentration dataset, (d) Mixed Liquor Suspended Solid (MLSS) dataset

\section{RESULTS METHOD}

Support Vector Machine (SVM) is first introduced by Vapnik [24] which is based on the idea of structural risk management. SVM is basically a supervised learning method that is capable in generating predictive models and used for classifiying unseen patterns into their categories. Quite recently, SVM method has been extended in solving function estimation and regression problem. The algorithm of SVM method is briefly explained in the following section.

\subsection{Support vector machine}

The basic theory of Support Vector Machine (SVM) regression function is expressed as in (1) [9].

$$
y=f(x)=\omega \cdot x+b
$$

where $y$ is scalar output, $\omega, x$ is a weight vector, $x$ is multivariate input and $b$ is bias. By introducing slack variables, $\xi_{i}$ and $\xi_{i}^{*}$, the SVM model can be expressed by (2) and (3).

$$
\begin{array}{cc}
\text { Minimize } & \phi(\omega)=\frac{1}{2}\|\omega\|^{2}-C \sum_{i=1}^{n}\left(\xi_{i}-\xi_{i}^{*}\right) \cdot C \geq 0 \\
\text { Subject to: } & \left\{\begin{array}{c}
y-w x-b \leq \varepsilon+\xi_{i}, \\
w x-b-y \leq \varepsilon+\xi_{i}^{*}, i=1,2, \ldots, \\
\xi_{i}, \xi_{i}^{*} \geq 0,
\end{array}\right.
\end{array}
$$

The new predicted SVM for each $x^{\prime}$ is determined by evaluating (4)

$$
y^{\prime}=\sum_{i=1}^{L}\left(\alpha_{i}^{+}-\alpha_{i}^{=}\right) x_{i} \cdot x^{\prime}+b
$$

\subsubsection{Validation of SVM model}

The division processes of normalized sequencing batch reactor data are determined by three folds cross validation concept based on two folds for training and onefold for testing. The cross validation is to access the performance of the SVM model besides checking the suitable parameters of cost of penalty, and gamma, value to be used. The best model is selected based on the accuracy performance correlation (R2) and mean square error (MSE) value. Figure 3 shows the flowchart of SVM method. 


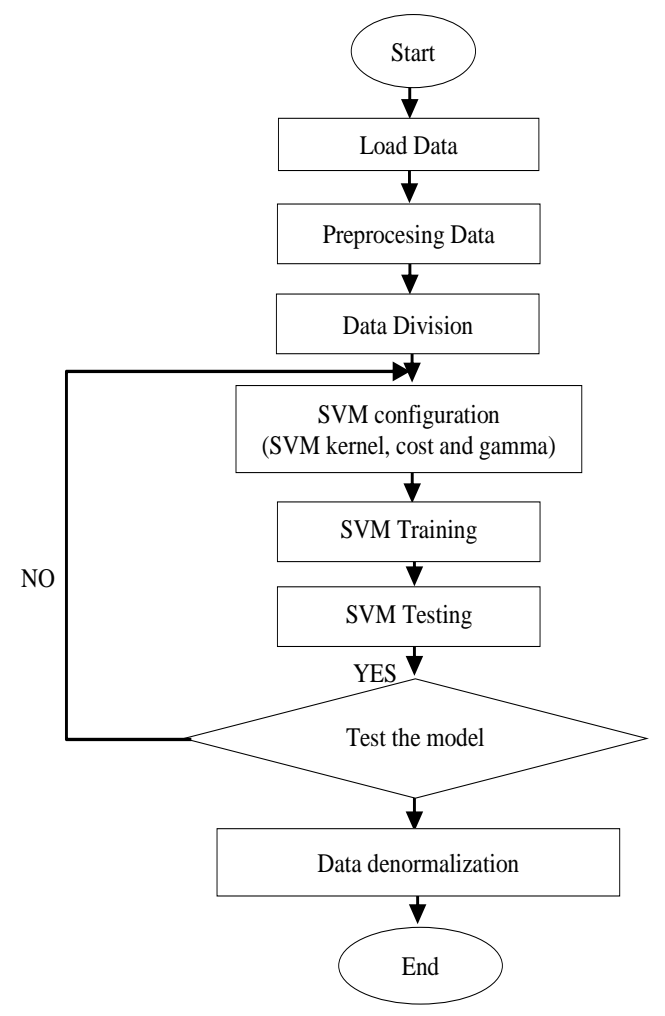

Figure 3. Flow chart of SVM prediction model

\subsection{Feed-forward neural network}

Basic theory of Feed forward neural network (FFNN) is the combination multilayer network. First layer is where the input from external input received. The second layer will be connected from the previous layer neurons. This layer will continue until it reached the final output layer neuron. Figure 4 shows the basic FFNN structure.

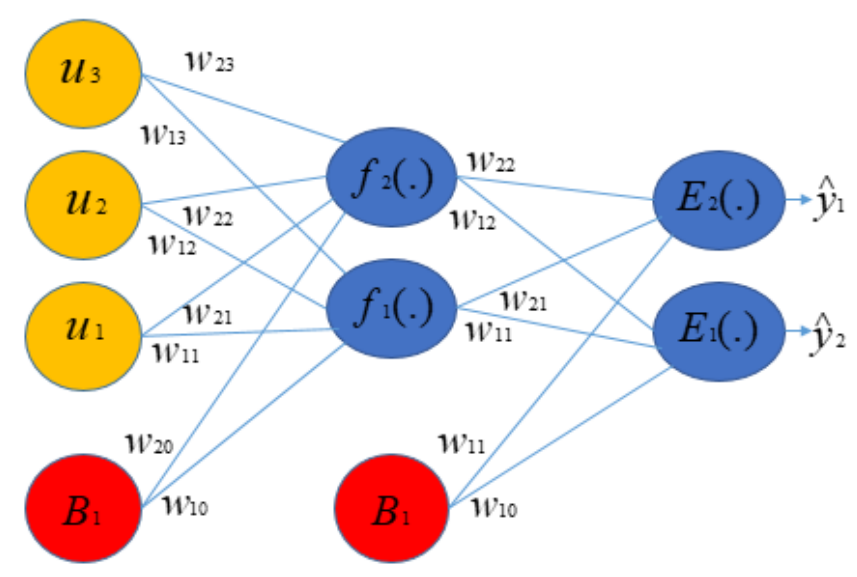

Figure 4. Feed forward neural network structure

This network is represented by (5);

$$
y^{\prime}=\sum_{i=1}^{L}\left(\alpha_{i}^{+}-\alpha_{i}^{=}\right) x_{i} \cdot x^{\prime}+b
$$


Where $y_{i}(t)$ is the model output of FFNN, $F_{i}$ is a function of the network, $W_{i j}$ and $w_{i j}$ is the connection layer of biases and weight, $\varphi$ is input layer. Figure 5 shows the flowchart of FFNN method.

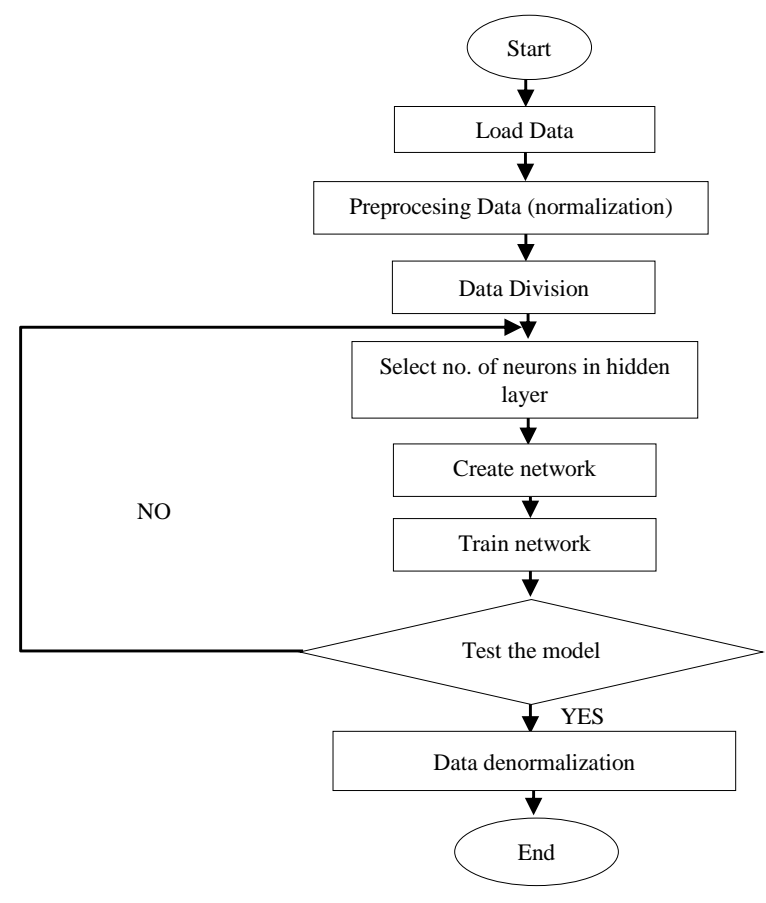

Figure 5. Flow chart of SVM prediction model

\section{RESULT AND DISCUSSION}

In general, both SVM and ANN techniques can provide good results in training and testing, although in terms of accuracy of the models, SVM is preferable to ANN. The model prediction of AGS system considered in this work are COD, TP, AN and AN variables. Figure 6(a) shows the COD evaluation results during training dataset. The SVM method predicted a good results as it achived $99.99 \%$ for R2 and 0.001 for MSE while FFNN achived slightly lower than SVM which is $92.52 \%$ for R2 and 0.0047 for MSE. In COD estimation for testing data, SVM gives higher accurate result compared with FFNN method for all evaluation criterion. Figure 6(b) shows the comparison prediction result performace of COD for testing where SVM result score are $99.84 \%$ and 0.0 while FFNN score are $96.44 \%$ and 0.0021 for R2 and MSE value, respectively. The COD estimation for overall training and testing performance result as shown in Table 1.

Results for TP training data shows good accuracy performance for both model techniques. The R2 result for both model SVM and FFNN achieved more than $90 \%$. The R2 value for SVM and FFNN are $96.34 \%$ and $93.36 \%$ while MSE are 0.0017 and 0.0047 respectively. Figure 7(a) shows the comparison graph of TP for both model SVM and FFNN. The testing result for TP estimation shows higher prediction value for SVM model compared to FFNN model. The scored of both testing value for R2 are $99.73 \%$ and $95.46 \%$ while MSE results are 0.0003 and 0.0044 respectively. Figure 7(b) shows the testing data result. The performance results of TP for both training and testing are shown in Table 2. 


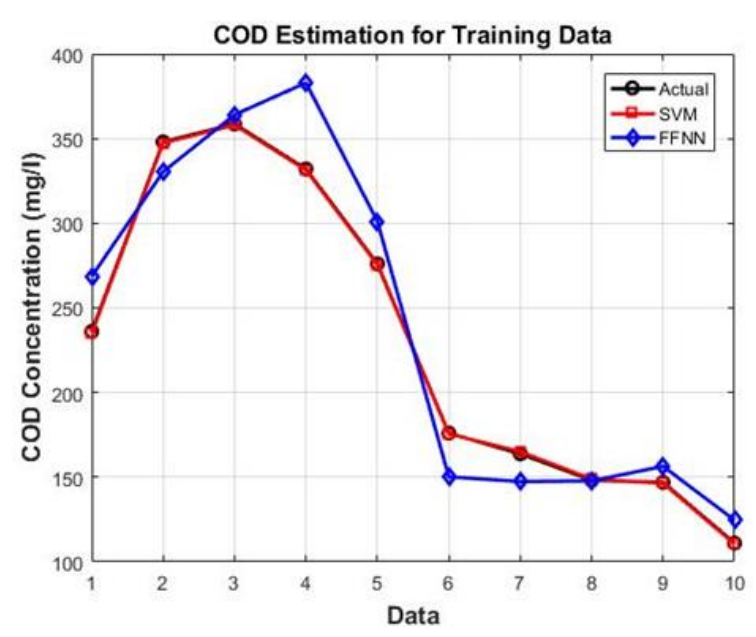

(a)

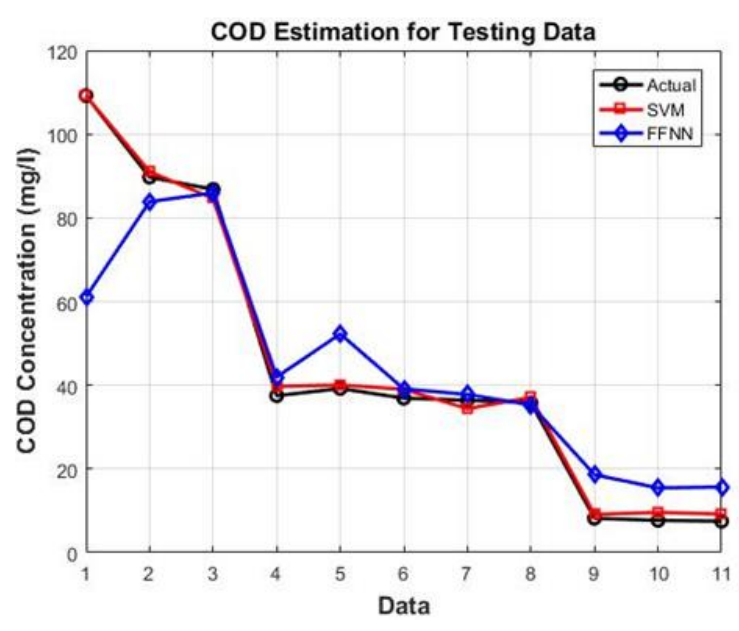

(b)

Figure 6. (a) COD training result for SVM and ANN, and (b) COD testing result for SVM and ANN

Table 1 . COD model evaluation for training and testing

\begin{tabular}{ccccc}
\hline Model & \multicolumn{2}{c}{ Training } & \multicolumn{2}{c}{ Testing } \\
\hline & $\mathrm{R}^{2}(\%)$ & MSE & $\mathrm{R}^{2}(\%)$ & MSE \\
SVM & 99.99 & 0.0001 & 99.84 & 0.0000 \\
FFNN & 92.53 & 0.0047 & 96.44 & 0.0021 \\
\hline
\end{tabular}

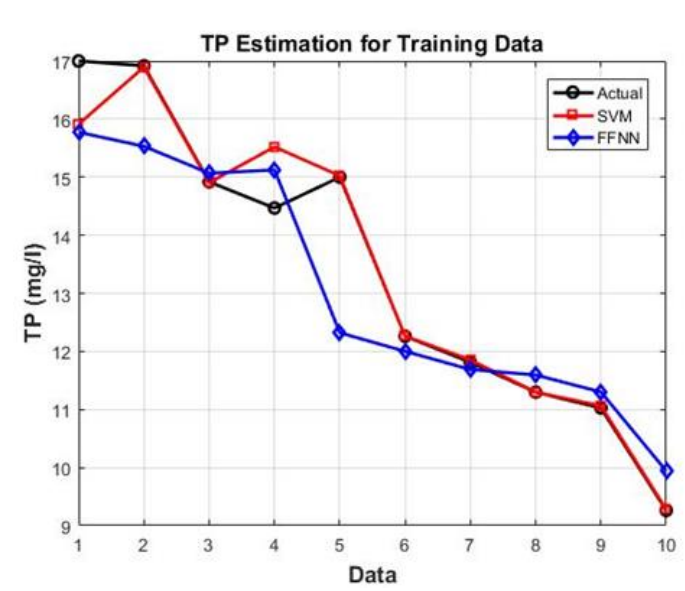

(a)
Table 2. TP testing result for SVM and ANN

\begin{tabular}{ccccc}
\hline Model & \multicolumn{2}{c}{ Training } & \multicolumn{2}{c}{ Testing } \\
\hline & $\mathrm{R}^{2}(\%)$ & MSE & $\mathrm{R}^{2}(\%)$ & MSE \\
SVM & 96.34 & 0.0017 & 99.73 & 0.0003 \\
FFNN & 93.36 & 0.0047 & 95.46 & 0.0044 \\
\hline
\end{tabular}

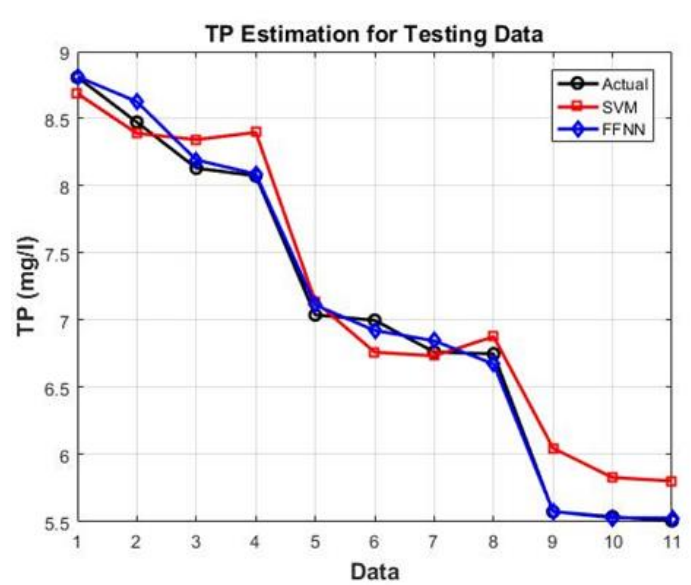

(b)

Figure 7. (a) TP training result for SVM and ANN, (b) TP testing result for SVM and ANN

The TN results show good prediction result for SVM method compared to FFNN method. The R2 for training and testing of SVM method both achieved $90 \%$ above and MSE 0.0006 while for FFNN method, the R2 and MSE is slightly lower prediction with $75.31 \%$ and 0.0022 respectively. During testing, R2 and MSE achieved 95.69 and 0.002. Figure 8(a) and 8(b) show an overall performance for both methods. Table 3 presents the performance comparison of both methods. 


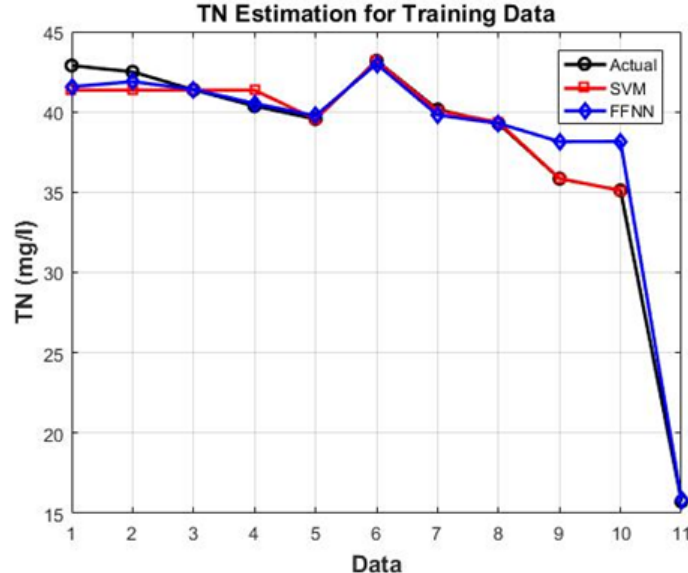

(a)

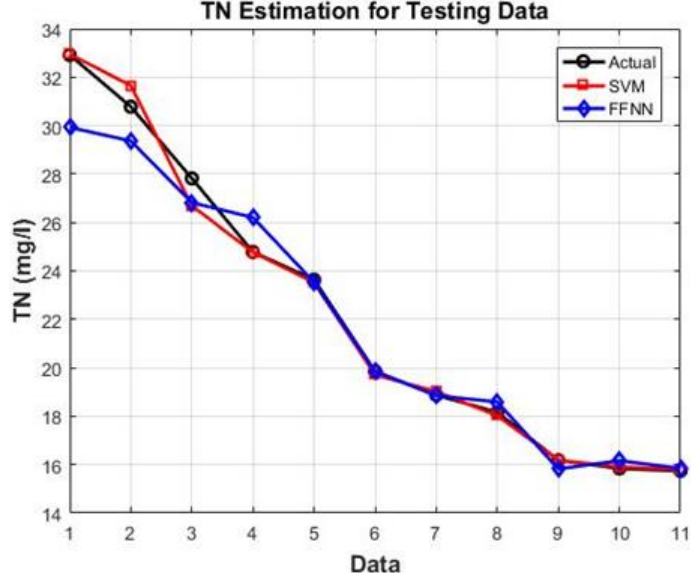

(b)

Figure 8. (a) TN training result for SVM and ANN, (b) TN testing result for SVM and ANN

The result for AN estimation shows acceptable accuracy from both methods. During AN testing, SVM methods achieved $95.09 \%$ and 0.0036 for R2 and MSE value, respectively. For FFNN, the achieved R2 and MSE are $86.06 \%$ and 0.0101 , respectively. During testing, SVM achieved the R2 of $90.12 \%$ and MSE of 0.0012 which is more accurate than FFNN with R2 of $80.48 \%$ and MSE of 0.0023. Figure 9(a) and 9(b) show the AN output response between SVM and FFNN during training and testing. Table 4 indicates an overall performance for both methods.

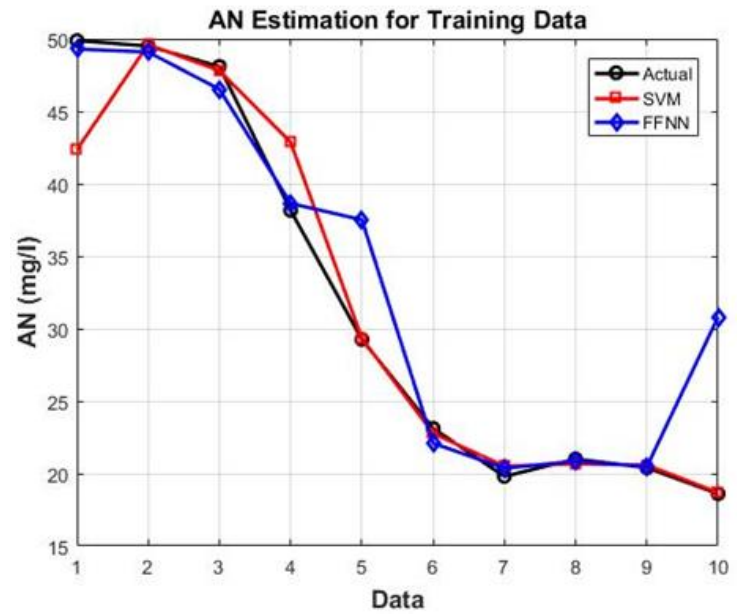

(a)

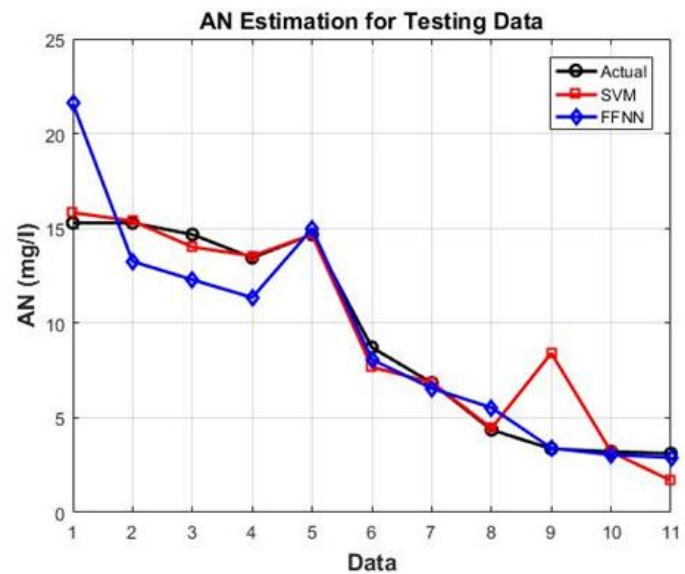

(b)

Figure 9. (a) AN training result for SVM and ANN, (b) AN testing result for SVM and ANN

Table 3. TN testing result for SVM and ANN

\begin{tabular}{ccccc}
\hline Model & \multicolumn{2}{c}{ Training } & \multicolumn{2}{c}{ Testing } \\
\hline & $\mathrm{R}^{2}(\%)$ & MSE & $\mathrm{R}^{2}(\%)$ & MSE \\
SVM & 96.34 & 0.0017 & 99.45 & 0.0003 \\
FFNN & 93.36 & 0.0047 & 95.69 & 0.0020
\end{tabular}

\section{CONCLUSION}

This paper presents the modelling techniques for aerobic granular sludge using SVM and compared with feed-forward neural network (FFNN). From the simulation results, it can be observed that the training 
result using SVM produces better accuracy of model for the prediction at the temperature of $50^{\circ} \mathrm{C}$. This provide an evident that SVM can give better prediction compared to FFNN model for limited training data. To improve the computational time of SVM parameters in future, external optimizations such as gravitational search algorithm (GSA), and Particle Swarm Optimization (PSO) can be considered.

\section{REFERENCES}

[1] M. Henze, C. Grady Jr, and W. Gujer, "Marais GvR and Matsuo T. Activated Sludge Model No. 1. IWA STR No 1", ed: IWA Publishing, London, UK, 1987.

[2] N. Prakash, S. Manikandan, L. Govindarajan, and V. Vijayagopal, "Prediction of biosorption efficiency for the removal of copper (II) using artificial neural networks," Journal of Hazardous Materials, vol. 152, pp. 1268-1275, 2008.

[3] C. Zhou, Z. Wang, Y. Liang, and J. Yao, "Study on the control of pore sizes of membranes using chemical methods Part II. Optimization factors for preparation of membranes," Desalination, vol. 225, pp. 123-138, 2008.

[4] Ł. Jedliński and J. Jonak, "Early fault detection in gearboxes based on support vector machines and multilayer perceptron with a continuous wavelet transform," Applied Soft Computing, vol. 30, pp. 636-641, 2015.

[5] K. Boussu, C. Vandecasteele, and B. Van der Bruggen, "Relation between membrane characteristics and performance in nanofiltration," Journal of Membrane Science, vol. 310, pp. 51-65, 2008.

[6] X. Xi, Y. Cui, Z. Wang, J. Qian, J. Wang, L. Yang, et al., "Study of dead-end microfiltration features in sequencing batch reactor (SBR) by optimized neural networks," Desalination, vol. 272, pp. 27-35, 2011.

[7] V. Vapnik, The nature of statistical learning theory: Springer Science \& Business Media, 2013.

[8] Z.-y. Jia, J.-w. Ma, F.-j. Wang, and W. Liu, "Hybrid of simulated annealing and SVM for hydraulic valve characteristics prediction," Expert Systems with Applications, vol. 38, pp. 8030-8036, 2011.

[9] V. Vapnik, S. E. Golowich, and A. Smola, "Support vector method for function approximation, regression estimation, and signal processing," Advances in neural information processing systems, pp. 281-287, 1997.

[10] B. Schölkopf, C. Burges, and A. Smola, "Advances in Kernel Methods-Support Vector LearningMIT Press," Cambridge, MA, 1999.

[11] B. Scholkopf et al., "Comparing support vector machines with Gaussian kernels to radial basis function classifiers," in IEEE Transactions on Signal Processing, vol. 45, no. 11, pp. 2758-2765, Nov. 1997.

[12] U. Thissen, R. Van Brakel, A. De Weijer, W. Melssen, and L. Buydens, "Using support vector machines for time series prediction," Chemometrics and intelligent laboratory systems, vol. 69, pp. 35-49, 2003.

[13] A. J. Smola and B. Schölkopf, "A tutorial on support vector regression," Statistics and computing, vol. 14, pp. 199-222, 2004.

[14] C. J. Burges, "A tutorial on support vector machines for pattern recognition," Data mining and knowledge discovery, vol. 2, pp. 121-167, 1998.

[15] C.-C. Chang and C.-J. Lin, "LIBSVM: a library for support vector machines," ACM transactions on intelligent systems and technology (TIST), vol. 2, p. 27, 2011.

[16] W. S. Noble, "What is a support vector machine?," Nature biotechnology, vol. 24, p. 1565, 2006.

[17] Y. Xu, S. Zomer, and R. G. Brereton, "Support vector machines: a recent method for classification in chemometrics," Critical Reviews in Analytical Chemistry, vol. 36, pp. 177-188, 2006.

[18] S. Liang and Z. Sun, "Sketch retrieval and relevance feedback with biased SVM classification," Pattern Recognition Letters, vol. 29, pp. 1733-1741, 2008.

[19] E. Gumus, N. Kilic, A. Sertbas, and O. N. Ucan, "Evaluation of face recognition techniques using PCA, wavelets and SVM," Expert Systems with Applications, vol. 37, pp. 6404-6408, 2010.

[20] M. Gao, J. Tian and J. Li, "The Study of Membrane Fouling Modeling Method Based on Support Vector Machine for Sewage Treatment Membrane Bioreactor," 2007 2nd IEEE Conference on Industrial Electronics and Applications, Harbin, 2007, pp. 1393-1398.

[21] W. Li-juan and C. Chao-bo, "Support Vector Machine Applying in the Prediction of Effluent Quality of Sewage Treatment Plant with Cyclic Activated Sludge System Process," 2008 IEEE International Symposium on Knowledge Acquisition and Modeling Workshop, Wuhan, 2008, pp. 647-650.

[22] X. Xi, Z. Wang, J. Zhang, Y. Zhou, N. Chen, L. Shi, et al., "Prediction of impacts of fabrication conditions on the filtration performance of homemade $\mathrm{VC}-\mathrm{co}-\mathrm{VAc}-\mathrm{OH}$ microfiltration membrane by Support Vector Machine (SVM)," Desalination and Water Treatment, vol. 51, pp. 3970-3978, 2013.

[23] C. Shang, X. Gao, F. Yang, W. Lyu, and D. Huang, "A comparative study on improved DPLS soft sensor models applied to a crude distillation unit," IFAC-PapersOnLine, vol. 48, pp. 234-239, 2015.

[24] C. Cai, W. Wang, L. Sun, and Y. Chen, "Protein function classification via support vector machine approach," Mathematical biosciences, vol. 185, pp. 111-122, 2003. 


\section{BIOGRAPHIES OF AUTHORS}
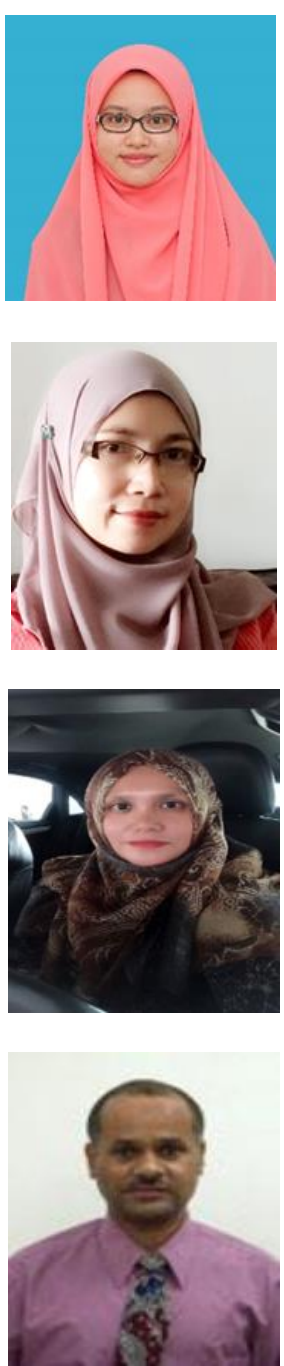

Nur Sakinah Ahmad Yasmin is Master student in School of Electrical Engineering, Faculty of Engineering, Universiti Teknologi Malaysia, Skudai, Johor. Received her bachelor degree in Electrical Control \& Instrument in Universiti Teknologi Malaysia, Skudai, Johor. Her area of interest is applying data mining technique in Wastewater Treatment fields and application

Dr. Norhaliza Abdul Wahab is currently an Associate Professor at Universiti Teknologi Malaysia (UTM). She is currently the Director for Control and Mechatronic Engineering at the School of Electrical Engineering, UTM. She completed her PhD in Electrical Engineering majoring in Control in July 2009. She is actively involved in researching and teaching in the field of industrial process control. Her expertise is in modelling and control of industrial process plant. Recently she has worked primarily on different types of domestic and industrial water and wastewater treatment technology towards optimization and energy saving system.

Dr. Aznah Nor Anuar is currently a Senior Lecturer at Department of Environmental and Green Technology, Malaysia-Japan International Institute of Technology, Universiti Teknologi Malaysia (UTM). She completed her PhD in Environmental Engineering and her thesis is on Development of Aerobic Granular Sludge Technology for Hot Climate Countries, graduated in July 2009. Her recent research interests in Environmental Engineering are in the area of wastewater-related disaster risk management, microbial granulation for wastewater treatment and low cost-green-sustainable technology for water and wastewater management. At present, she has published more than 15 journals, 15 expert reports and 70 proceedings that covers topic of her research interests

Mustafa Bob has completed his $\mathrm{PhD}$ at the age of 35 years from The Ohio State University and he was a postdoctoral fellow with the National Research Council (NRC) in USA. He is currently an assistant professor at Taibah University in Saudi Arabia. He has published more than 10 papers in reputed journals and has been serving as a reviewer for a number of reputed journals. 